\title{
Role of MR Enterography in Evaluation of Disease Activity in Pediatric Crohn's Disease: Correlation between MR Enterography and Pediatric Crohn's Disease Activity Index Scores
} \author{
Suja Rajan² \\ 1Department of Pediatric Radiology, Kanchi Kamakoti CHILDS Trust \\ Hospital, Chennai, India \\ ${ }^{2}$ Department of Radiodiagnosis, Government Stanley Medical \\ College, Chennai, India \\ ${ }^{3}$ Department of Pediatric Gastroenterologist, Kanchi Kamakoti \\ CHILDS Trust Hospital, Chennai, India
}

Shilpa Radhakrishnan ${ }^{1}$ Amarnath Chellathurai ${ }^{2}$ Srinivas Sankaranrayanan ${ }^{3}$ Dharani Sankar

Address for correspondence Amarnath Chellathurai, MD, FRCR, Department of Radiodiagnosis, Government Stanley Medical College, Chennai 60000, India (e-mail: amarrd02@yahoo.co.in).

\begin{abstract}
Keywords

- CD MRI index

- Crohn's disease

- MR enterography global score

Objectives The aim of the study was to assess the role of MR Enterography (MRE) in the diagnosis and follow-up of children with Crohn's disease (CD) and to correlate disease activity indices with known MRE features of active disease.

Methods This was a retrospective study including 24 patients (median age 11 years, 17 males) with clinically and histologically proven CD who underwent MRE. Two previously validated MRE scores-Magnetic Resonance Enterography Global Score (MEGS) and $C D$ MRI index (CDMI)-were calculated. A correlative analysis was made between the Pediatric Crohn's Disease Activity Index (PCDAI) score and MRE scores as well as individually with each MR variable. Comparison of both the MR scores was made between patients with different disease activity.

Results MEGS and PCDAl scores showed strong positive correlation ( $r=0.724$, $p=<0.001)$; CDMI and PCDAI scores showed moderate positive correlation ( $r=0.661$, $p=0.0004)$. There was statistically significant difference in the MR scores between patients grouped by clinical activity. Among individual MR variables, mural thickness and enhancement best predicted the disease activity.

Conclusions MRE-based scores and findings correlate with clinical activity in pediatric $C D$. Thereby, MRE can be considered a valuable tool in the management of $C D$, predicting disease activity and offering a potential alternative to endoscopy in monitoring patients during follow-up.
\end{abstract}

\section{Introduction}

Pediatric inflammatory bowel disease (PIBD) and Crohn's disease (CD) are not rare entities, with 20 to $30 \%$ of $C D$ presenting before age of 20 years. Symptoms of PIBD can be nonspecific including growth failure, malnutrition, delayed puberty, and so forth, other than gastrointestinal symptoms. Treatment goal in PIBD has moved beyond symptom control to mucosal healing and histological control. Thus, treatment strategy depends on accurate assessment of disease location, extent and activity. The gold standard for assessing mucosal
DOI https://doi.org/ 10.1055/s-0040-1701328 ISSN 2581-9933.
License terms

() (1) $\ominus \circledast$ 
activity is ileocolonoscopy. However, this being invasive and often limited to colon and terminal ileum, clinical scoring like Pediatric Crohn's Disease Activity Index (PCDAI) is often used, especially when reassessing while on treatment. ${ }^{1}$ These clinical indices have the disadvantage of being subjective and cumbersome to calculate. Magnetic resonance enterography (MRE) and magnetic resonance (MR) scoring give a better idea of the burden of disease including transmural and extraintestinal involvement and are increasingly used in diagnosis and follow-up of PIBD with good sensitivity and accuracy. The European Society for Pediatric Gastroenterology Hepatology and Nutrition (ESPGHAN) revised porto criteria recommend MRE as the imaging modality of choice in PIBD for diagnosis. ${ }^{2}$

Most often than not, MR assessment is usually subjective instead of there being quantitative measurements. Rimola et $\mathrm{al}^{4}$ proposed and validated the Magnetic Resonance Index of Activity (MaRIA) in adult inflammatory bowel disease (IBD) patients correlating it with endoscopic scoring. ${ }^{3}$ However, both endoscopy and MaRIA score take only mucosal findings into consideration. Moreover, the proposed MR protocol included colonic preparation and dynamic contrast assessment which may be cumbersome in children. Steward et al proposed the Crohn's Disease Activity Score (CDMI/ CDAS) which was a segmental MRI activity score validated against histological scoring, taking into account extraintestinal disease as well. ${ }^{5}$ Based on this score Makanyanga et al developed a global score (MEGS) and compared it with clinical markers of activity like fecal calprotectin, $\mathrm{C}$ reactive protein, and the Harvey Bradshaw index (HBI). ${ }^{6}$

All these MR scores have been shown to be good predictors of disease activity in adults. Though there are few studies in assessment of MR enterography in pediatric Crohn's disease, ${ }^{7,8}$ there are no studies in our knowledge that compare the usefulness and validation of these MR scores in pediatric population. The aim of this study is to correlate the MEGS and CDMI scores with clinical activity score (PCDAI) in children.

\section{Materials and Methods}

This was a single institutional retrospective analysis of patients with histologically proven $\mathrm{CD}$. All patients who were diagnosed with CD between January 2013 and December 2016, and underwent MRE either for diagnosis or follow-up were included.

\section{Patients}

The study included 24 patients who were referred for MRE by the pediatric gastroenterologist for any of the following indications: diagnosis, assessment of disease, evaluation of perianal disease, or response assessment. We had 7 girls and 17 boys with a mean age of 11 years at diagnosis. Patients with inadequate bowel distension and children who already underwent surgery for complications related to $C D$ were excluded because inadequate bowel distension would mask underlying lesion and it would be difficult to analyze the MRI features of the bowel wall in an operated segment.

\section{Clinical Assessment}

Clinical disease activity was assessed by calculating the PCDAI score by the same pediatric gastroenterologist. A PCDAI score of $\geq 30$ is considered indicative of moderate/severe disease.

\section{MR Enterography}

Imaging was performed on a $1.5 \mathrm{~T}$ system (Siemens, MAGNETOM AERA, Germany) with patients in supine position, after 4 to 6 hours of fasting. Oral preparation or bowel cleansing was not consistently used. Contrast images were acquired after administering $0.1 \mathrm{mmol} / \mathrm{kg}$ body weight of gadolinium contrast agent. Each imaging series consisted of breath hold (whenever possible) axial, coronal, sagittal single-shot fast spin echo T2-weighted sequences, axial and coronal steady state free precession sequences, and axial and coronal T1-weighted fat suppressed sequences before and after contrast administration. Diffusion-weighted imaging was not consistently performed ( $\mathbf{-}$ Table $\mathbf{1}$ ).

\section{Analyses of MR Enterography and Scoring}

The images were reviewed by two experienced radiologists blinded to the patient's clinical scores at the time of scans. In case of any discrepancies a consensus read was performed.

The following MR features were recorded: mural thickness (-Fig. 1), T2 signal intensity (-Fig. 2), degree and pattern of

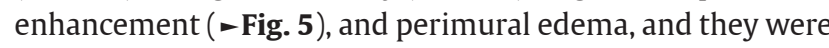
quantitatively scored (0-3) as described by Steward et al. ${ }^{5}$

Extramural findings like lymphadenopathy, comb sign (-Fig. 4), abscess, and perianal disease (-Fig. 3) were recorded as present or absent (-Figs. 1-5).

Two previously validated MRE scores, MEGS ${ }^{6}$ and CDMI, were calculated. The details of the score have been listed in the appendices.

Table 1 MR enterography parameters used in our study

\begin{tabular}{|l|l|l|l|}
\hline Parameter & HASTE & True FISP & VIBE \\
\hline Repetition time (ms) & 2,000 & 3.50 & 4.36 \\
\hline Echo time (ms) & 94 & 1.45 & 2 \\
\hline Flip angle $\left(^{\circ}\right)$ & 150 & 50 & 10 \\
\hline Matrix (pixel) & $320 \times 81$ & $256 \times 100$ & $320 \times 75$ \\
\hline Section thickness (mm) & 3.5 & 3 & 3 \\
\hline Intersection gap (mm) & 0 & 0 & 0 \\
\hline Field of view & $300-350$ & $300-350$ & $300-350$ \\
\hline
\end{tabular}




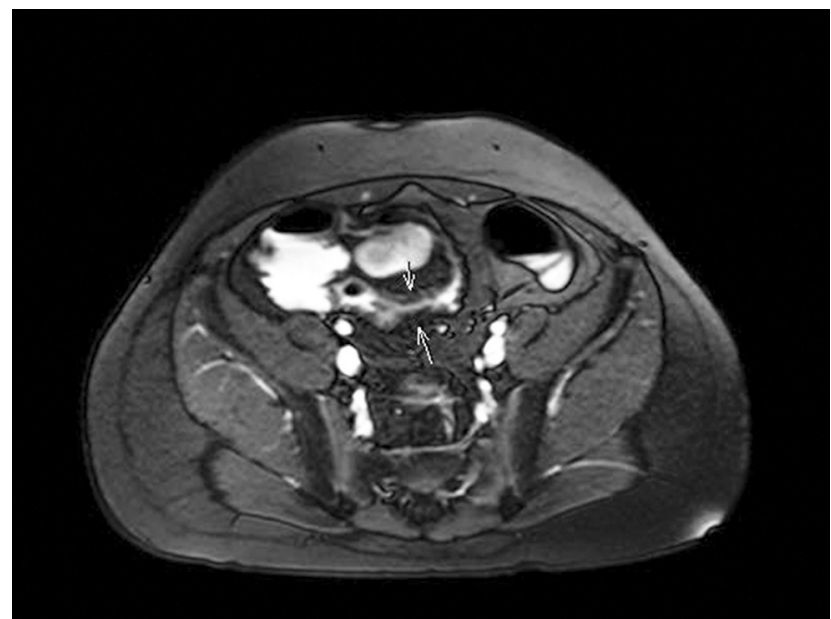

Fig. 1 T1 VIBE fat suppressed axial images shows wall thickening with mucosal enhancement pattern in the terminal ileum.

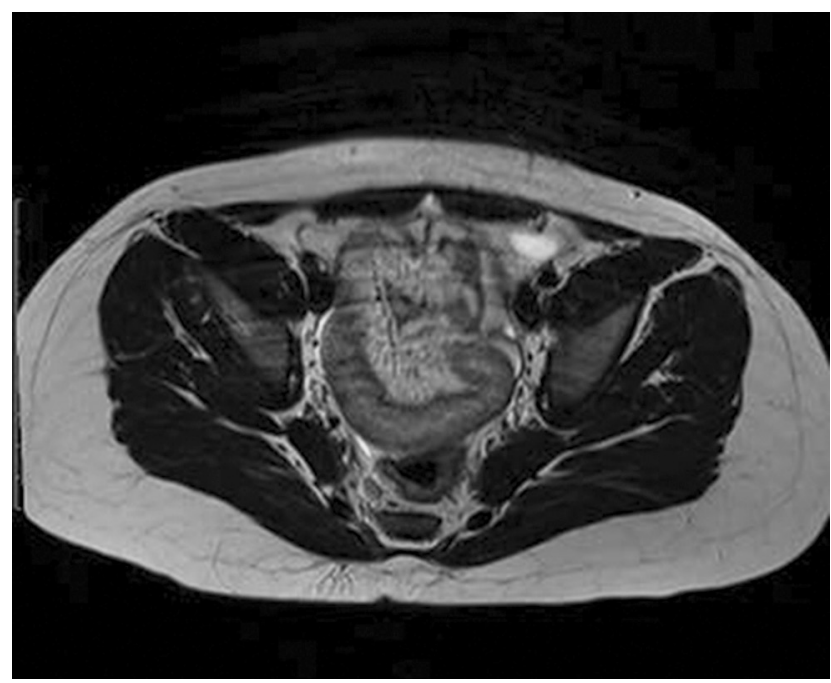

Fig. 2 T2 axial turbo spin echo sequence in a known case of Crohn's disease shows wall thickening with increased signal intensity of the involved ileal loop.

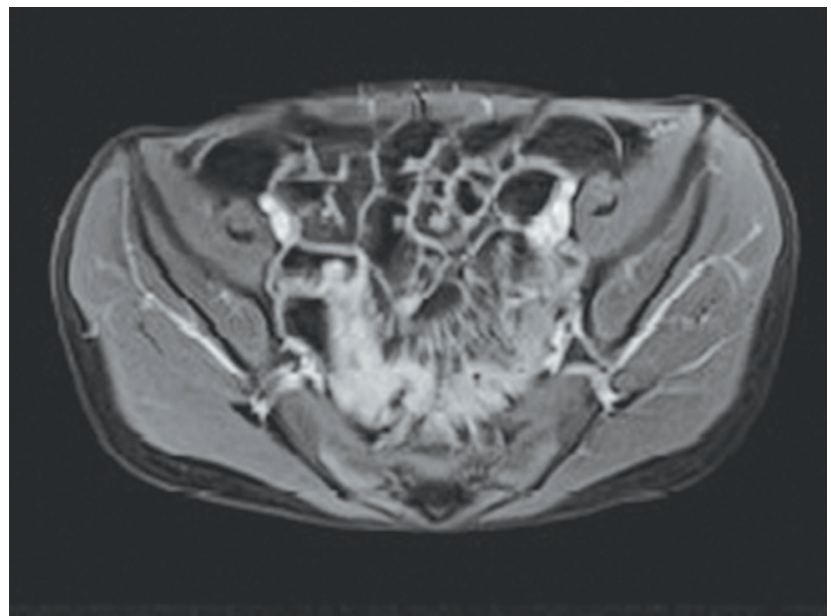

Fig. 4 T1 VIBE fat suppressed axial images of pelvis shows hypervascular appearance of the mesentery-comb sign.

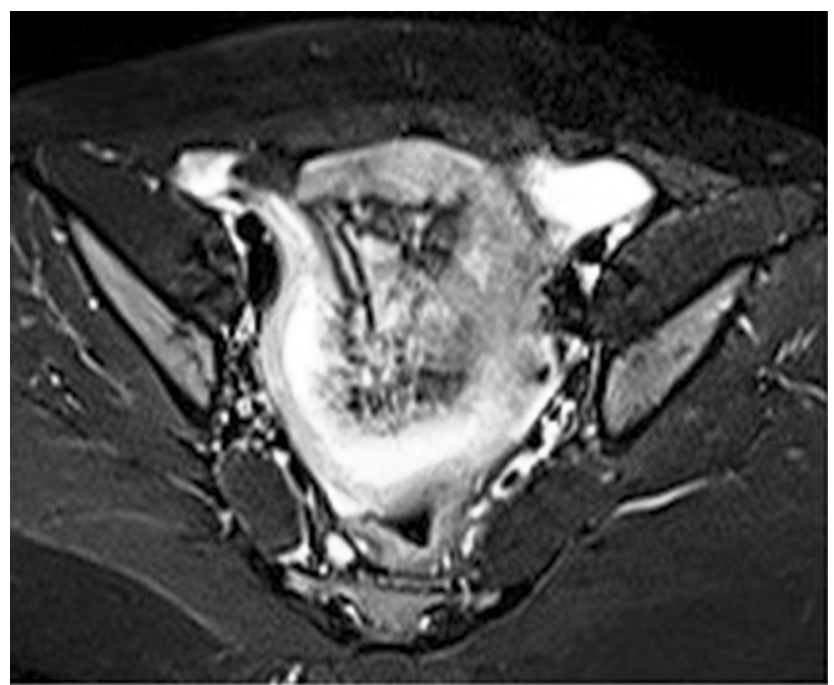

Fig. 5 T2 STIR axial image of pelvis in case of active crohn's disease shows layered pattern of increased signal intensity in a thickened ileal loop.
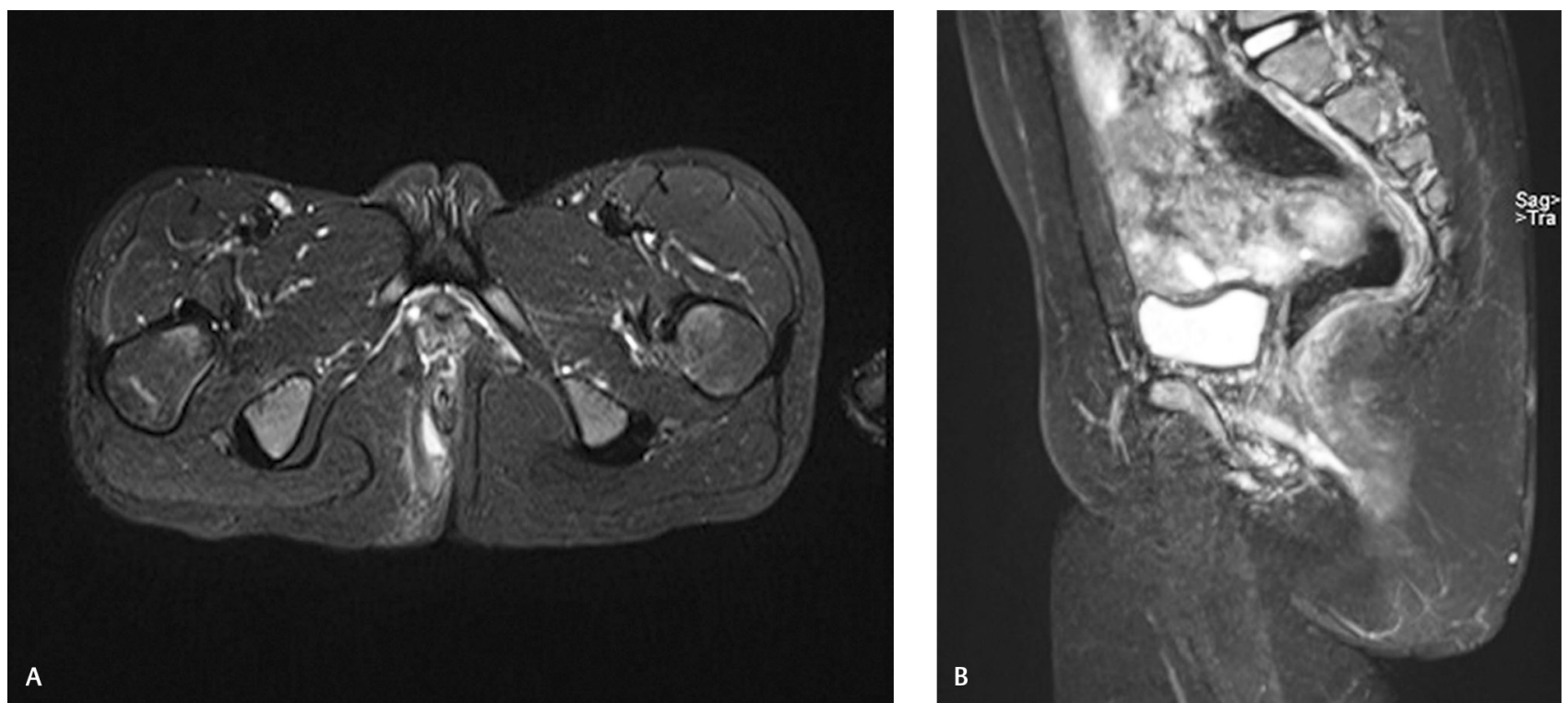

Fig. 3 (A, B) STIR axial and sagittal images of pelvis in a case of penetrating Crohn's disease shows fluid filled linear intersphincteric fistula on right side. 
For the purpose of calculating the MEGS score the bowel was divided into eight anatomical segments: jejunum; ileum; terminal ileum; ascending, transverse, descending, and sigmoid colons; and rectum. Individual segmental scores are added together, and then 5 points are added for presence of lymph nodes $\geq 10 \mathrm{~mm}$ (short-axis diameter), comb sign, fistula, or abscess. The length of the diseased segment was used to provide a multiplication factor (ranging from 1 to 2 ) for each segmental score. ${ }^{6}$

For each patient, the MEGS were then calculated as the sum of the scores for all intestinal segments.

\section{Data Analysis}

- Table 2 shows the demographics and clinical data of 24 patients included in the study.

\section{Results}

\section{MRI Features}

Fifteen had abnormal wall thickening defined as $>3 \mathrm{~mm}$, 7 patients (29.2\%) had mild (3-5 mm; - Fig. 1) thickening, 2 (8.3\%) had moderate $(5-7 \mathrm{~mm})$, and 6 (25\%) had marked (>7 mm) thickening.

High wall signal intensity on T2-weighted images ( - Fig. 2) was seen in 17 patients, of which $5(20.8 \%)$ were mild, 11 (45.8\%) moderate, and 1 (4.2\%) were marked.

Table 2 Clinical and demographic data

\begin{tabular}{|l|l|}
\hline Age in y median (range) & $11(5-17)$ \\
\hline Gender, $n(\%)$ & \\
\hline Male & $17(70.8 \%)$ \\
\hline Female & $7(29.2 \%)$ \\
\hline Disease location, $n$ (\%) & \\
\hline Isolated small bowel & $11(45.8 \%)$ \\
\hline Ileocolonic & $4(16.7 \%)$ \\
\hline Colonic & $3(12.5 \%)$ \\
\hline Terminal ileum & $2(8.3 \%)$ \\
\hline Associated with perianal fistula & $2(8.3 \%)$ \\
\hline PCDAl median (range) & $22.5(0-65)$ \\
\hline MEGS median (range) & $15.25(0-53)$ \\
\hline CDMI median (range) & $7.5(0-13)$ \\
\hline Extramural features & \\
\hline Lymph nodes & $10(41.7 \%)$ \\
\hline Comb sign & $12(54.2 \%)$ \\
\hline
\end{tabular}

All cases with abnormal wall thickening had mural enhancement of varying degrees.

Marked enhancement was seen in 9 (37.5\%) cases.

Enhancement pattern was recorded as mucosal $(n=5)$ transmural/homogenous $(n=9)$ and striated $(n=6)$.

PCDAI scores ranged from 0 to 65 (median 22.5) while the MEGS and CDMI scores ranged from 0 to 53 and 0 to 13 , respectively ( - Table $\mathbf{3}$ ).

-Figure 6A shows the correlation of MRE scores with PCDAI.The MEGS and PCDAI scores showed strong positive correlation $\left(r=0.724, p=<0.001, r^{2}=0.5237\right)$. CDMI and PCDAI scores showed strong positive correlation ( - Fig. $6 \mathrm{~B} ; r=0.661$, $\left.p=0.00043, r^{2}=0.4367\right)$. Individual MRI features were correlated with the PCDAI scores where wall thickness showed a strong correlation $(r=0.792, p=<0.001$; - Fig. 7A $)$, wall T2 signal showed a weak correlation $(r=0.499, p=0.02123)$ ( - Fig. 7B), and wall enhancement showed a moderate posi-

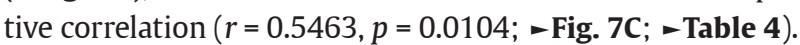

Pattern of enhancement showed a weak correlation $(r=0.394, p=0.07726)$. Extramural features like presence of lymph nodes and comb sign $(r=0.338)$ showed weak correlation to PCDAI score ( $\mathbf{- T a b l e ~} \mathbf{5})$.

Patients were grouped according to their clinical activity (PCDAI scores) into group A score $<30(n=16$, having mild activity) and group B score 30 and above ( $n=8$, having moderate to severe disease; - Table 6 ).

The MEGS and CDMI scores ranged from 0 to 28 (median $=14.50$ ) and 0 to 10 (median $=4.50$ ), respectively, in group A and from 19 to 59 (median $=20.5$ ) and 7 to 13 (mean =

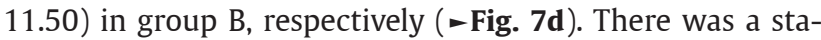
tistically significant difference in the scores between the two groups by using both $t$-test as well as Mann-Whitney test (nonparametric) $(p<0.05)$.
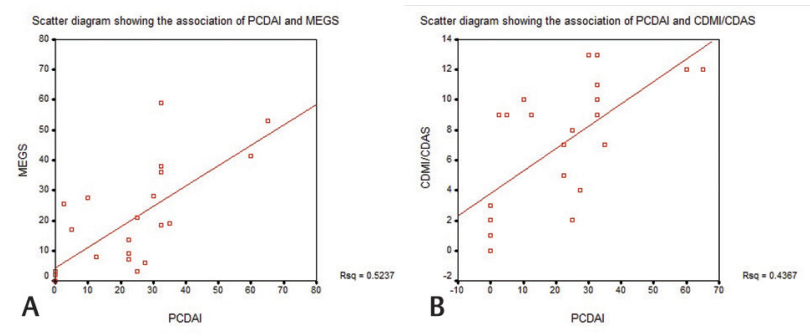

Fig. 6 Correlation between PCDAI with MEGS and CDMI scores. (A) Scatter diagram showing strong positive correlation between the MEGS and PCDAI scores. (B) Scatter diagram illustrating CDMI and PCDAl scores with strong positive correlation $(r=0.661, p=0.00043$, $\left.r^{2}=0.4367\right)$.

Table 3 Detailed report of PCDAI, MEGS, and CDMI scores

\begin{tabular}{|l|l|l|l|l|l|}
\hline $\begin{array}{l}\text { Descriptive } \\
\text { statistics }\end{array}$ & No. & Minimum & Maximum & Mean & $\begin{array}{l}\text { Standard } \\
\text { deviation }\end{array}$ \\
\hline Age in years & 24 & 5 & 17 & 11.05 & 2.958 \\
\hline PCDAI & 24 & 0 & 65.0 & 20.833 & 18.9627 \\
\hline MEGS & 24 & 0 & 53.0 & 17.881 & 15.1821 \\
\hline CDMI/CDAS & 24 & 0 & 13 & 7.00 & 4.012 \\
\hline
\end{tabular}

Abbreviations: CDMI/CDAS, Crohn's Disease Activity Score; MEGS, Magnetic Resonance Enterography Global Score; PCDAI, Pediatric Crohn's Disease Activity Index. 


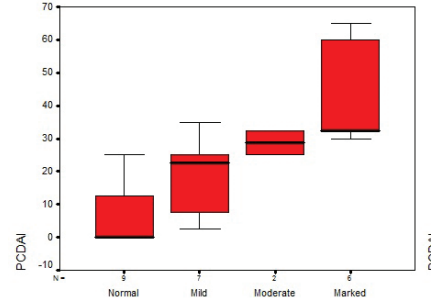

A Thick score

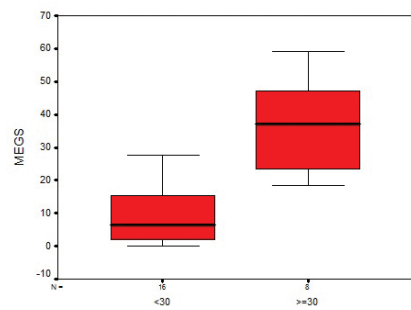

D

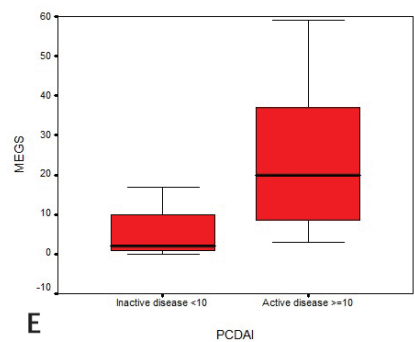

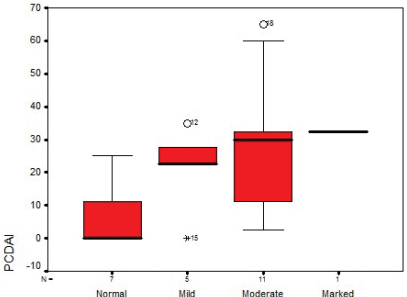

B

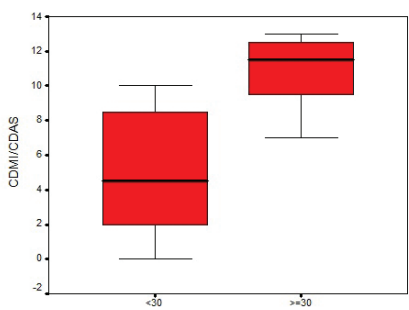

PCDAI

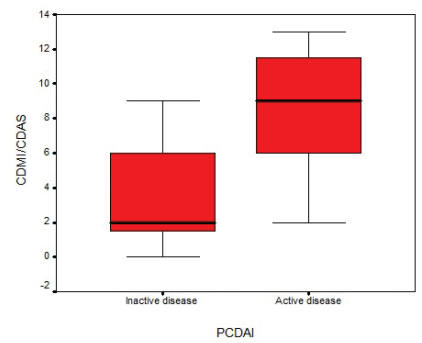

Fig. 7 Correlation between MRE features and PCDAl scores: (A) Wall thickness showed strong correlation $(r=0.792, p=<0.001)$. (B) Wall T2 signal showed weak correlation $(r=0.499, p=0.02123)$. (C) Wall enhancement showed moderate positive correlation $(r=0.5463, p=0.0104)$. Correlation between PCDAI groups with MEGS and PCDAI scores: (D) Statistically significant difference in the scores between the two groups by using both $t$-test as well as Mann-Whitney test (nonparametric) $(p<0.05)$. (E) Correlation between groups $C$ and D with MEGS and PCDAI scores.

Table 4 Correlation between PCDAI with MEGS and CDMI scores

\begin{tabular}{|c|c|c|c|c|}
\hline & & PCDAI & MEGS & CDMI/CDAS \\
\hline \multirow[t]{3}{*}{ PCDAI } & Pearson correlation & 1 & $0.782^{\mathrm{a}}$ & $0.637^{\mathrm{a}}$ \\
\hline & Sig. (2-tailed) & NA & 0.000 & 0.002 \\
\hline & $N$ & 24 & 24 & 24 \\
\hline \multirow[t]{3}{*}{ MEGS } & Pearson correlation & $0.782^{\mathrm{a}}$ & 1 & $0.891^{\mathrm{a}}$ \\
\hline & Sig. (2-tailed) & 0.000 & NA & 0.000 \\
\hline & $N$ & 24 & 24 & 24 \\
\hline \multirow[t]{3}{*}{ CDMI/CDAS } & Pearson correlation & $0.637^{a}$ & $0.891^{\mathrm{a}}$ & 1 \\
\hline & Sig. (2-tailed) & 0.002 & 0.000 & NA \\
\hline & $N$ & 24 & 24 & 21 \\
\hline
\end{tabular}

Abbreviations: NA, not applicable; PCDAI Pediatric Crohn's Disease Activity Index; MEGS, Magnetic Resonance Enterography Global Score; CDMI, Crohn's Disease MRI Index; CDAS, Crohn's Disease Activity Score

aCorrelation is significant at the 0.01 level (2-tailed).

Similarly, patients were grouped into two groups group C, PCDAI 1 to 10 (inactive disease [ $n=8]$ ), and group D, PCDAI 10 and above (active disease of varying severity $[n=16]$ ). The MEGS and CDMI scores ranged from 0 to $26($ median $=13.5)$ and 0 to 9 (median $=5$ ), respectively, in group $C$ and from 3 to 59 (median $=28.5)$ and 2 to 13 (median $=6.5$ ), respectively, in group D (-Table 6).
The difference in MR scores between these two groups was also statistically significant. There were two cases where the PCDAI was less than 10 but MRE score showed activity. This patient continued to have active disease on treatment without remission and went on to have hemicolectomy. 
Table 5 Correlation between MRE features and PCDAI scores. Comprehensive correlation between PCDAI and MRE features

\begin{tabular}{|l|l|}
\hline MRE features & Pearson correlation coefficient $(\boldsymbol{r})$ \\
\hline Wall thickness & $0.792^{\mathrm{a}}$ \\
\hline T2 wall signal & $0.499^{\mathrm{b}}$ \\
\hline Wall enhancement & $0.546^{\mathrm{b}}$ \\
\hline Pattern of enhancement & 0.394 \\
\hline Lymph nodes & -0.082 \\
\hline Comb sign & 0.338 \\
\hline
\end{tabular}

Abbreviations: MRE, magnetic resonance enterography; PCDAI, Pediatric Crohn's Disease Activity Index

${ }^{a}$ Correlation is significant at the 0.01 level (2-tailed).

${ }^{\mathrm{b}}$ Correlation is significant at the 0.05 level (2-tailed).

Table 6 Correlation between PCDAI groups with MEGS and PCDAI scores. Groups divided according to PCDAI score

\begin{tabular}{|l|l|l|l|l|}
\hline & Group A & Group B & Group C & Group D \\
\hline PCDAI & $<30$ mild activity & $\begin{array}{l}>30 \text { moderate to severe } \\
\text { activity }\end{array}$ & $<10$ inactive disease & $>10$ active disease \\
\hline No. of studies $(\boldsymbol{n})$ & 16 & 8 & 8 & 16 \\
\hline
\end{tabular}

Abbreviations: MEGS, Magnetic Resonance Enterography Global Score; PCDAI, Pediatric Crohn's Disease Activity Index.

\section{Discussion}

As pediatric IBD cases are being diagnosed more and more, the need for an accurate objective test to assess disease activity is paramount. This is even more important when reassessing patients on treatment. Treatment goal of $\mathrm{CD}$ is to achieve the best possible histological control and hence evaluation of mucosal activity is important. Clinical activity indices have the drawback of relying on subjective symptoms. Endoscopy remains the gold standard but is invasive and poorly tolerated in children with the added disadvantage of not being able to assess the proximal small bowel at all times. ${ }^{9}$ Besides, extraintestinal disease is not assessed and information about actual disease burden is limited. Even though MRE is shown to have good performance in evaluating PIBD, the description and interpretation of MRE findings is not standardized. ${ }^{7.8}$ There is limited agreement in the available literature as to which MRI feature best correlates with disease activity. The best way to do this would be a universally validated MRI score which incorporates different intestinal and extraintestinal findings and is simple enough to be incorporated by radiologists in their routine reporting. Various MR based scoring systems described have been validated only in adults so far. There have not been many studies comparing known MRE scores with PCDAI scores.

The main focus of our study was to correlate two previously validated MRE scores with clinical activity index. The global MEGS score has already been validated by Makanyanga et al against fecal calprotectin and CRP in adults. ${ }^{3}$ However, they found no significant correlation with HBI clinical score. When Steward et al developed the CDMI score they showed significant correlation between the MR score and histological acute inflammatory scores (AIS). ${ }^{5}$
Our main finding was that there is strong positive correlation between both CDMI and MEGS with PCDAI score. However MEGS has higher correlation compared to CDMI (-Table 4).

When we grouped our patients based on clinical activity, we found that the MR scores among the groups were also correlating. This proves that MRE based scoring is as good as clinical scoring especially when following up patients on treatment to predict disease activity and to confirm disease remission.

\section{Limitations and Recommendations}

Our study population was limited. However, this study can still serve as a preliminary analysis with reproducible results.

We correlated the MR scores only with clinical scoring which has a subjective nature. There was no correlation with any histopathological scores.

Another limitation of our study was that bowel preparation and MR protocols were not consistent within the study population. Similarly in cases which had no or inadequate bowel preparation, inadequate distension may have masked small mucosal lesions or ulcerations.

This emphasizes the need for a large multicenter study with standardized protocols and consistent imaging parameters, bowel preparation, and sequences.

\section{Keypoints}

Studies comparing PCDAI scores and MRE scores are lacking in pediatric population. MRE scoring of cronh's disease can help predict disease activity compliment clinical indices potentially replacing endoscopy.

\section{Conflict of Interest}

None declared. 


\section{References}

1 Hyams JS, Ferry GD, Mandel FS, et al. Development and validation of a pediatric Crohn's disease activity index. J Pediatr Gastroenterol Nutr 1991;12(4):439-447

2 Levine A, Koletzko S, Turner D, et al; European Society of Pediatric Gastroenterology, Hepatology, and Nutrition. ESPGHAN revised porto criteria for the diagnosis of inflammatory bowel disease in children and adolescents. J Pediatr Gastroenterol Nutr 2014;58(6):795-806

3 Rimola J, Rodriguez S, García-Bosch O, et al. Magnetic resonance for assessment of disease activity and severity in ileocolonic Crohn's disease. Gut 2009;58(8):1113-1120

4 Rimola J, Ordás I, Rodriguez S, et al. Magnetic resonance imaging for evaluation of Crohn's disease: validation of parameters of severity and quantitative index of activity. Inflamm Bowel Dis 2011;17(8):1759-1768

5 Steward MJ, Punwani S, Proctor I, et al. Non-perforating small bowel Crohn's disease assessed by MRI enterography: derivation and histopathological validation of an MR-based activity index. Eur J Radiol 2012;81(9):2080-2088

6 Makanyanga JC, Pendsé D, Dikaios N, et al. Evaluation of Crohn's disease activity: initial validation of a magnetic resonance enterography global score (MEGS) against faecal calprotectin. Eur Radiol 2014;24(2):277-287

7 Dillman JR, Ladino-Torres MF, Adler J, et al. Comparison of MR enterography and histopathology in the evaluation of pediatric Crohn disease. Pediatr Radiol 2011;41(12):1552-1558

8 Horsthuis K, de Ridder L, Smets AM, et al. Magnetic resonance enterography for suspected inflammatory bowel disease in a pediatric population. J Pediatr Gastroenterol Nutr 2010;51(5):603-609

9 Gee MS, Nimkin K, Hsu M, et al. Prospective evaluation of MR enterography as the primary imaging modality for pediatric Crohn disease assessment. AJR Am J Roentgenol 2011;197(1):224-231

10 Harvey RF, Bradshaw JM. A simple index of Crohn's-disease activity. Lancet 1980;1(8167):514 